\title{
On the Classification of Imbalanced Datasets
}

\author{
Arun Kumar M.N \\ Research Scholar \\ Dept. of Electronics and Communication Engg. \\ P.E.S. College of Engneering \\ Mandya, India
}

\author{
H.S. Sheshadri \\ Professor \\ Dept. of Electronics and Communication Engg. \\ P.E.S. College of Enginering \\ Mandya, India
}

\begin{abstract}
In recent research the classifications of imbalanced data sets have received considerable attention. It is natural that due to the class imbalance the classifier tends to favour majority class. In this paper we investigate the performance of different methods for handling data imbalance in the microcalcification classification which is a classical example for data imbalance problem. Micro calcifications are very tiny deposits of calcium that appear as small bright spots in the mammogram. Classification of microcalcification clusters from mammograms plays an important role in computer-aided diagnosis for early detection of breast cancer. In this paper, we review in brief the state of the art techniques in the framework of imbalanced data sets and investigate the performance of different methods for microcalcification classification.
\end{abstract}

\section{Keywords}

Classification, Microcalcification, Imbalanced data sets, Mammography.

\section{INTRODUCTION}

Imbalanced data classification often arises in many practical applications. Many classification approaches are developed by assuming the underlying training set is evenly distributed. However, those approaches are faced with a severe bias problem when the training set is a highly imbalanced distribution. There are many real-world problems those are faced with severe problem of learning for imbalanced class.

The imbalanced data $[1,2,3,4,5,6]$ cause classifiers to perform poorly on the minority class. When the data are highly imbalanced many existing methods tend to misclassify the minority class. The imbalanced problem has attracted much attention in machine learning community. There are many real-world applications that are faced with class imbalance problem. Some examples for these applications include fault diagnosis, anomaly detection, medical diagnosis, e-mail foldering, face recognition etc.

Breast cancer $[54,55,56,57,58,59,60,61]$ is the most frequently occurring cancer and one of the leading causes of death among women. Early diagnosis and subsequent treatment can significantly improve the chance of survival for patients with breast cancer. Mammography is the most effective method for the detection of early breast cancer. Mammograms are among the most difficult radiological images to interpret by radiologists. Its images are often very poor in contrast. It can show different features and patterns depending on breast anatomy and tissues density. Radiologists do not detect all breast cancers that are retrospectively detected on the mammograms.

Mammogram facilitates a manner of automated screening which acts as a "second reader" to the physician. There are two major processes involved in the development of techniques that perform automatic analysis and interpretation of breast mammogram: Computer-Aided Detection (CADe) and Computer-Aided Diagnosis (CADx). Detection is the ability to identify potential abnormalities, such as microcalcification, masses, and architectural distortions. Diagnosis is the ability to characterize or classify a detected abnormal entity (architectural distortion, masses, microcalcification clusters) as being either benign or malignant. Figure 1 shows the three important abnormalities which are to be detected by any CAD system on digital mammograms. There are a series of tasks done before CADe algorithms can identify suspicious regions in a mammogram. These include: pre-processing, segmentation, feature extraction, and classification.

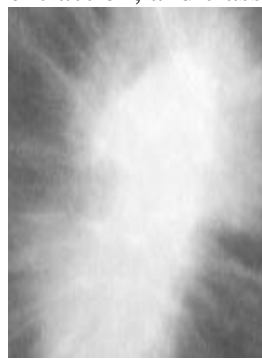

a) Spiculations

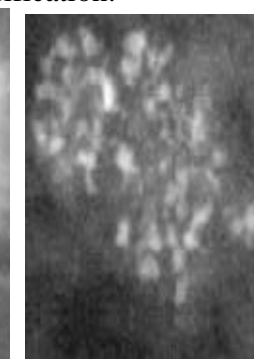

b) Calcifications c) Circumscribed lesions Fig. 1 Important Abnormalities
Classification of real (potential) microcalcification in a mammogram is a classical example for class imbalance problem. In this paper we review novel classification methods for imbalance data problems and investigate the ability of different methods for handling data imbalance in the microcalcification classification. Paper is organized as follows. Related works that deals with class imbalance problem and microcalcification classification are discussed in section 2 . Investigation of the performance of different methods in the microcalcification classification along with pre-processing method is described in section 3 . Conclusion is drawn in section 4 .

\section{LITERATURE SURVEY}

Many methods for imbalanced data for binary class and multi class problems are developed. Methods $[1,2,3,4,5,6,7,8,9$, $10,11,12,13,14,15,16,17,18,19]$ are summarized in table 1. Many other methods developed are briefed out in the following paragraphs.

Vladimir Nikulin et.al. proposed [20] an ensemble based method on a large number of balanced random sets. The proposed method is general and may be implemented in conjunction with different base-learners. They have concluded further improvement that can be achieved using more advanced pre-processing technique. Albert Orriols-Puig et.al. [21] investigated the capabilities of evolutionary on-line rulebased systems which extracts knowledge from imbalanced data. Proposed methods are flexible that can be adapted to detect class imbalance cases and find suitable models. A combination of random forest based techniques and sampling 
methods to identify the potential buyers are proposed [22] by Jie Gu. Proposed method showed its favourable performance improvement in terms of the area under the ROC. Bhavani Raskutti [23] reported methods on balancing training data which has two classes of interest in heavily unbalanced proportions. They focused on the case of supervised learning with support vector machines. Gang $\mathrm{Wu}$ et.al. proposed the class-boundary alignment algorithm [24] to augment SVMs to deal with imbalanced training-data problems posed by applications like image retrieval, video surveillance, and gene profiling. Mikel Galar et.al. proposed [25] taxonomy for ensemble-based methods to address the class imbalance where each proposal can be categorized depending on the inner ensemble methodology in which it is based. They developed a thorough empirical comparison by other approaches. In the paper [26] authors reviewed the existing methods for solving the class imbalance problem. They have discussed the various metrics used to evaluate the performance of classifiers. By combining both unsupervised and supervised learning they introduced a new approach to solve the class imbalance problem.

In the paper [27] authors discussed the problem of class or cost imbalance. By outlining some of the trends Nitesh V. Chawla et.al. attempted to chart out the progress in related areas of learning from imbalanced data sets. They discussed many issues that stimulate new directions and solutions. These directions and solutions lead to both theoretical insight and practical applications. Suzan Koknar-Tezel et.al. presented an innovative approach [28] that augments the minority class by adding synthetic points in distance spaces. The proposed approach used Support Vector Machines for classification. Haibo He et.al. discussed [29] new challenges and critical problem in the knowledge discovery and the imbalanced learning problem. Their discussions of the fundamental nature of the imbalanced learning problem, the state-of-the-art solutions used to address critical problem, and the several major assessment techniques used to evaluate the problem served as a comprehensive resource for existing and future knowledge discovery and data engineering researchers and practitioners. Seyda Ertekin et.al. developed an efficient method [30] for active selection of informative instances from a randomly picked small pool of samples. In the proposed method they have maintained the same or achieving even higher g-means values by using less number of training instances in the SVM model. Xing-Ming Zhao in their article [31] presents a new technique for protein classification with imbalanced data. They proposed a new algorithm to overcome the imbalanced problem in protein classification. J. Burez, D et.al. used more appropriate [32] evaluation metrics. These metrics are AUC and lift. They investigated the increase in performance of random and advanced under-sampling and two specific modelling techniques. Baek Hwan Cho et.al. accurately predicted the onset of diabetic nephropathy. They effectively applied various machine learning techniques [33] to irregular and unbalanced diabetes dataset. Those methods include support vector machine (SVM) classification and feature selection methods. For evaluating imbalanced dataset authors introduced a novel measure [34] as a better alternative. They provide a theoretical background for the new evaluation technique that is designed to cope with cost biases. Sireesha Rodda et.al. in their paper [35] presented a method in learning from multi-class imbalanced dataset. The proposed method does not break dataset into a series of binary class datasets. For estimating the quality of classification rule they introduced a new measure called normalized strength score. Many methods [ 36, 37, 38, 39, 40, 41, 42, 43, 44, 45, 46, 47, $48,49,50,51,52,53]$ are developed for the microcalcification classification. Many methods proposed in the literature results in less classification accuracy in terms of $\mathrm{Az}$ (Area under ROC curve). The reason is the difficulty in dealing with imbalanced data set. Many machine learning algorithm fail in dealing with imbalanced data set. We will investigate the performance of different methods for the classification of microcalcification

\section{EXPERIMENTAL ANALYSIS}

Data collection of imbalanced datasets related to microcalcification for experimental analysis is extracted from MIAS database and this involves some steps. These steps are discussed in the following 2 subsections. Last subsection summarizes the performance of different methods for microcalcification classification.

\subsection{Preprocessing}

We have applied some preprocessing steps to remove the artifacts, and noises in the mammograms. Also the pectoral muscle is segmented to reduce the processing area. Median filter of size $3 \mathrm{X} 3$ is used to remove the noise. It is the nonlinear filter used to remove the impulse noise from an image. To remove the artifacts the mammogram is opened using a suitable structuring element, and then it is reconstructed. It is followed by thresholding the difference image with value 102. Morphological operators are applied to smooth irregularities and Sobel edge detector is used to detect the edge. Detected breast contour is superimposed on original image. Figure 2 shows the results of these steps for a MIAS image.

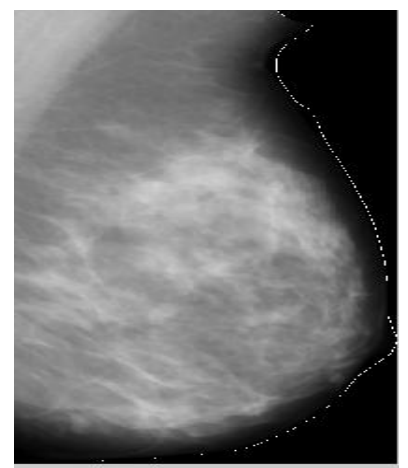

Fig 2. Breast contour superimposed on the original image Segmentation of pectoral muscle uses wavelet decomposition (Daubechies Wavelet) of fourth level and edge detection using Canny filter. Figure 3 shows the result of these steps in a MIAS image.

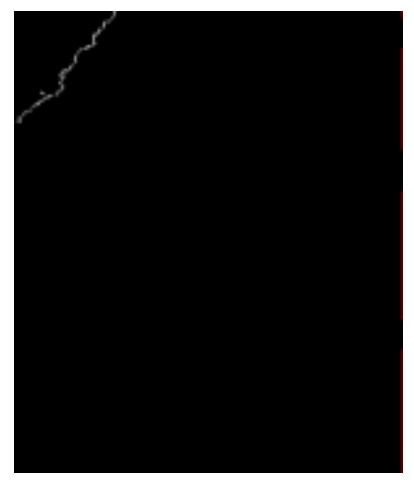

Fig 3. Pectoral muscle identified in the above image Identified pectoral muscle edge is superimposed on original image. For more details of our work refer [62]. Then binary images are created from each segmented image that is intended for helping automatic cropping procedure so the image will contain only the ROI. 


\subsection{Detection of Signals (Potential Micro calcification)}

This data collection phase uses only 22 MIAS images preprocessed in the previous phase since these images contain the microcalcification inside the boundaries of breast. To detect the signals, which are called as the potential microcalcification, construct a DoG filters and apply sequentially to the images obtained from previous phase by varying the binarization threshold. The points obtained after the application of each filter is added to the points obtained previously. The remaining DoG filter is used in the same way to the points obtained. These points are selected as the signals (potential microcalcification) according to the region area, gray level, and gray gradient. The resultant signals are preclassified into microcalcification and notmicrocalcification according to the available truth information. Only $0.4 \%$ of signals are microcalcification and $99.6 \%$ are not. We employed approach as in [63] for these steps. Because of this imbalanced distribution of classes data sampling is necessary. 35 features are extracted from each potential microcalcification related to their contrast and shape. Features are passed through a feature selection process and 5 relevant features are extracted.

\subsection{Investigation of different methods}

The main objective of the study is to evaluate the performance of the different classifiers. For data sampling we used SMOTE, Locally linear embedding, and four versions of SMOTE + Complementary Neural Network (CMTNN). In order to demonstrate that the proposed sampling technique can assist classification of imbalanced data, several classification algorithms are used. They are ANN, SVM, Bayesian Networks, and k-NN. The results of classification are evaluated and compared in terms of performance. Table 2 shows the comparison results.

\section{CONCLUSION}

There are many real-world applications, where uneven distribution of data patterns is very common. In these cases number of training samples of a minority class is much smaller compared to other majority classes. Microcalcification classification is one classical example for imbalanced data problem. In this paper, we review in brief the state of the art techniques in the framework of imbalanced data sets, and investigate the performance of different methods for handling data imbalance in the microcalcification classification. A range of alternative classifiers are selected and datasets are prepared or sampled in order to assess the performance. Future research work can be focused on medical images with intractable geometric complexity in data classification.

Table 1. Methods on class imbalanced data problems

\begin{tabular}{|c|c|c|c|c|}
\hline Method used & Dataset used & $\begin{array}{c}\text { Other } \\
\text { methods taken } \\
\text { for analysis }\end{array}$ & Remarks & Future work \\
\hline $\begin{array}{l}\text { [1] Bi-class problem } \\
\text { Based on SVM and } \\
\text { backward pruning } \\
\text { technique }\end{array}$ & $\begin{array}{l}1484 \text { samples collected from } \\
\text { SWISS-PROT } \\
\quad \text { and } \\
\text { Hyperspectral/Polarimetric target } \\
\text { detection }\end{array}$ & $\begin{array}{l}\text { SVM-based } \\
\text { weighting method }\end{array}$ & $\begin{array}{l}\text { Proposed method outperforms } \\
\text { SVM-based weighting method. }\end{array}$ & $\begin{array}{l}\text { Employ other pruning } \\
\text { method such as branch and } \\
\text { bound. }\end{array}$ \\
\hline $\begin{array}{l}\text { [2] Bi-class problem } \\
\text { Linear embedding } \\
\text { algorithm incorporated } \\
\text { on conventional } \\
\text { SMOTE algorithm }\end{array}$ & $\begin{array}{l}\text { Three datasets are collected from } \\
\text { several chest X-ray image } \\
\text { databases. Sample size of three } \\
\text { data sets is } 1180,1530 \text {, and } 1164 \\
\text { with minority classes of } .038 \% \text {, } \\
0.029 \% \text { and } 0.042 \% \\
\text { respectively. }\end{array}$ & SMOTE algorithm & $\begin{array}{l}\text { Proposed method outperforms } \\
\text { conventional SMOTE } \\
\text { algorithm }\end{array}$ & $\begin{array}{l}\text { Application of proposed } \\
\text { method on other medical } \\
\text { images with intractable } \\
\text { geometric complexity in } \\
\text { data classification. }\end{array}$ \\
\hline $\begin{array}{l}\text { [3] Bi-class problem } \\
\text { Adopted two methods. } \\
\text { 1. [Cost-sensitive] } \\
\text { Random Forest } \\
\text { 2. Sampling technique } \\
\text { + Ensemble idea }\end{array}$ & $\begin{array}{l}\text { Oil, Mammography, Satimage, } \\
\text { Hypothyroid, Euthyroid, KDD } \\
\text { thrombin of size 937, 11183, } \\
6435,2520,2640, \text { and } 2543 \\
\text { respectively. Percentage of } \\
\text { Minority class is } 4.4,2.3,9.7,4.8, \\
\text { 9.1, and 7.6 respectively. }\end{array}$ & $\begin{array}{l}\text { SHRINK, SMOTE, } \\
\text { and SMOTEBoost }\end{array}$ & $\begin{array}{l}\begin{array}{l}\text { Proposed } \\
\text { outperform }\end{array} \\
\text { SMOTE, AND SMOTEBoost. }\end{array} \begin{array}{r}\text { methods } \\
\text { SHRINK, }\end{array}$ & $\begin{array}{l}\text { Performance of the two } \\
\text { proposed methods under } \\
\text { label noise. }\end{array}$ \\
\hline $\begin{array}{l}\text { [4] Bi-class problem } \\
\text { Kernel-Based Two- } \\
\text { Class Classifier using } \\
\text { orthogonal forward } \\
\text { selection }\end{array}$ & $\begin{array}{l}\text { Synthetic data, Pima Indian } \\
\text { diabetes data, Haberman data, } \\
\text { ADI data, Satimage data }\end{array}$ & $\begin{array}{l}\text { 1-NN, 3-NN, SVM, } \\
\text { SUPANOVA, } \\
\text { RIPPER, SMOTE, } \\
\text { SMOTE-Boost, } \\
\text { BRF, WRF }\end{array}$ & $\begin{array}{l}\text { Proposed method outperforms } \\
\text { all other method. }\end{array}$ & $\begin{array}{l}\text { Underlying assumption of } \\
\text { the procedure may no } \\
\text { longer be suitable for } \\
\text { imbalanced data sets. So } \\
\text { research on the suitability } \\
\text { of the procedure over } \\
\text { imbalanced data set is } \\
\text { necessary }\end{array}$ \\
\hline $\begin{array}{l}\text { [5] Bi-class problem } \\
\text { SVM-WEIGHT } \\
\text { GSVM-RU } \\
\text { SVM-SMOTE } \\
\text { SVM-RANDU }\end{array}$ & $\begin{array}{l}\text { Oil, Mammography, Satimage, } \\
\text { Abalone (19 vs. other), Abalone } \\
\text { (9 vs. } 18) \text {, Yeast (ME2 vs.other), } \\
\text { Yeast (CYT vs. POX) }\end{array}$ & $\begin{array}{ll}\text { WRF, } & \text { AdaCost, } \\
\text { KBA, } & \text { SMOTE- } \\
\text { Boost } & \end{array}$ & $\begin{array}{l}\text { GSVM-RU outperforms all } \\
\text { other classifier }\end{array}$ & $\begin{array}{l}\text { Future research can be } \\
\text { focused on comparison } \\
\text { study on other benchmarks } \\
\text { with highly imbalanced } \\
\text { data sets }\end{array}$ \\
\hline $\begin{array}{lr}\text { [6] Bi-class problem } \\
\text { Two different versions } \\
\text { of } & \text { infinitely } \\
\text { imbalanced } & \text { logistic } \\
\text { regression } & \text { with } \\
\text { Gaussian } \quad k=1 \quad \text { and } \\
k=10 .\end{array}$ & Landmine, and Watermine dataset & LR & IILR outperforms LR & $\begin{array}{l}\text { Make IILR approach } \\
\text { suitable for datasets in } \\
\text { which the data points of the } \\
\text { rare class do not cluster } \\
\text { tightly. Other future work } \\
\text { can be focused on other } \\
\text { applications with severe } \\
\text { class imbalance. }\end{array}$ \\
\hline $\begin{array}{l}\text { [7] Bi-class problem } \\
\text { Hierarchical fuzzy rule } \\
\text { based classification } \\
\text { system }\end{array}$ & $\begin{array}{l}22 \text { data sets with low imbalance } \\
\text { and } 22 \text { data sets with high } \\
\text { imbalance. For details refer [7]. }\end{array}$ & 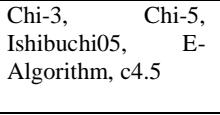 & $\begin{array}{l}\text { Proposed method outperforms } \\
\text { all other methods. }\end{array}$ & $\begin{array}{lccr}\text { Inclusion } & \text { of } & \text { a } & \text { multi- } \\
\text { objective } & \text { GA } & \text { for } & \text { rule } \\
\text { selection } & & & \end{array}$ \\
\hline $\begin{array}{l}\text { [8] Sampling based on } \\
\text { clustering (SBC), SBC }\end{array}$ & $\begin{array}{l}\begin{array}{l}\text { Synthetic dataset of } \\
\text { samples. }\end{array} \\
\text { sa, }\end{array}$ & $\begin{array}{l}\text { AT, RT, Nearmiss- } \\
2\end{array}$ & $\begin{array}{l}\text { For real dataset SBC } \\
\text { outperforms all other method }\end{array}$ & $\begin{array}{l}\text { Future research can be } \\
\text { focused on comparison }\end{array}$ \\
\hline
\end{tabular}




\begin{tabular}{|c|c|c|c|c|}
\hline $\begin{array}{l}\text { with Nearmiss-1, SBC } \\
\text { with Nearmiss-2, SBC } \\
\text { with Nearmiss3, SBC } \\
\text { with most distance, and } \\
\text { SBC with most far }\end{array}$ & $\begin{array}{l}\text { Census-Income datasets (real } \\
\text { dataset) of } 30,162 \text { samples. } 80 \% \\
\text { used for training and } 20 \% \text { used for } \\
\text { testing. } \\
\text { Overdue detection database (real } \\
\text { dataset) of } 62,309 \text { for training and } \\
63,532 \text { for testing. }\end{array}$ & & $\begin{array}{l}\text { For synthetic dataset SBC and } \\
\text { RT is most suited. }\end{array}$ & $\begin{array}{l}\text { study on other benchmarks } \\
\text { with highly imbalanced } \\
\text { data sets. }\end{array}$ \\
\hline $\begin{array}{l}\text { [10] Bi-class problem } \\
\text { Information } \\
\text { granulation based data } \\
\text { mining }\end{array}$ & $\begin{array}{l}\text { Two balanced datasets and } \\
\text { unbalanced datasets from UCI } \\
\text { machine learning repository }\end{array}$ & $\mathrm{NN}(\mathrm{BP})$ & $\begin{array}{l}\text { Proposed method outperforms } \\
\text { NN. }\end{array}$ & $\begin{array}{l}\text { To reduce the data } \\
\text { dimension } \\
\text { sophisticated tool can be } \\
\text { used. }\end{array}$ \\
\hline $\begin{array}{l}\text { [12] Bi-class problem } \\
\text { Combination of } \\
\text { supervised learning and } \\
\text { unsupervised clustering } \\
\text { to handle imbalanced } \\
\text { data set. }\end{array}$ & $\begin{array}{l}\text { Liver, Hepatitis, Pima diabetes, } \\
\text { and Wisconsin }\end{array}$ & RPROP & $\begin{array}{l}\text { Modified RPROP (Mod- } \\
\text { RPROP) has higher values of } \\
\text { G-mean and F-measure than } \\
\text { RPROP. But similar } \\
\text { classification rate for both. }\end{array}$ & $\begin{array}{l}\text { Future research can be } \\
\text { focused on highly } \\
\text { imbalanced data sets of } \\
\text { very less imbalanced ratio < } \\
0.1\end{array}$ \\
\hline $\begin{array}{l}\text { [13] Bi-class problem } \\
\text { Four combination of } \\
\text { SMOTE and } \\
\text { complementary neural } \\
\text { network (CMTNN) to } \\
\text { handle problem of } \\
\text { classifying imbalanced } \\
\text { data. } \\
\text { Three classifiers used } \\
\text { are ANN, k-NN, and } \\
\text { SVM }\end{array}$ & $\begin{array}{l}\text { Pima Indian diabetes data, } \\
\text { German credit data, } \\
\text { Haberman's survival data, SPECT } \\
\text { Heart data. }\end{array}$ & $\begin{array}{l}\text { ENN, Tomek links, } \\
\text { and SMOTE to } \\
\text { handle problem of } \\
\text { classifying } \\
\text { imbalanced data. }\end{array}$ & $\begin{array}{l}\text { For ANN classifier, four } \\
\text { combination of SMOTE and } \\
\text { CMTNN outperforms others. } \\
\text { For SVM classifier } \\
\text { Tomeklinks and one } \\
\text { combination } \\
\text { (SMOTE+CMTNN) } \\
\text { outperform other. } \\
\begin{array}{l}\text { For k-NN classifier, two } \\
\text { combination of SMOTE + } \\
\text { CMTNN }\end{array}\end{array}$ & $\begin{array}{l}\text { The number of instances } \\
\text { removed by the proposed } \\
\text { method is a major issue in } \\
\text { the classification accuracy } \\
\text { of the imbalanced data and } \\
\text { this issue has a future } \\
\text { scope. }\end{array}$ \\
\hline $\begin{array}{lr}15] \text { Bi-class problem } \\
\begin{array}{l}\text { Clustering } \\
\text { classification }\end{array} & \text { based } \\
\text { SUMO) } & \text { CLU- }\end{array}$ & $\begin{array}{lcc}\text { Datasets } & \text { from } & \text { MediaMill } \\
\text { Challenge Problem } & \end{array}$ & $\begin{array}{l}\text { SUMO, NB, NN, 3- } \\
\text { NN, Ada, DTree, } \\
\text { MP }\end{array}$ & $\begin{array}{l}\text { CLU-SUMO outperforms all } \\
\text { other methods }\end{array}$ & $\begin{array}{l}\text { To avoid the over fitting } \\
\text { problem experiments } \\
\text { pertain to the influence of } \\
\text { the cluster size K of the } \\
\text { proposed framework should } \\
\text { be conducted }\end{array}$ \\
\hline $\begin{array}{l}\text { [16] Bi-class problem } \\
\text { Active Learning } \\
\text { Heuristic (SALH) + } \\
\text { Wilcoxon-Mann- } \\
\text { Whitney (WMW) }\end{array}$ & $\begin{array}{l}6 \text { unbalanced datasets from UCI } \\
\text { repository } \\
\text { Abalone, Thyroid, Opt.Digits, } \\
\text { Solar Flare, Adult, Liver }\end{array}$ & $\begin{array}{l}\text { C4.5, Naïve Bayes, } \\
\text { Canonical GP, GP } \\
\text { under SALH }\end{array}$ & $\begin{array}{l}\text { SALH+WWW outperforms all } \\
\text { other method }\end{array}$ & $\begin{array}{l}\text { Future work can be focused } \\
\text { on the significance of } \\
\text { fitness function in GP } \\
\text { classifier }\end{array}$ \\
\hline $\begin{array}{l}\text { [17] Bi-class problem } \\
\text { k-Nearest Neighbor } \\
\text { Approach ( } 1-\mathrm{NN}, 3- \\
\mathrm{NN}, 5-\mathrm{NN}, 7-\mathrm{NN}, 9- \\
\mathrm{NN})\end{array}$ & $\begin{array}{llll}\begin{array}{l}\text { Dataset } \\
\text { abstracts }\end{array} & \text { of } & 300 & \text { MEDLINE } \\
\end{array}$ & C5.0 & $\begin{array}{l}\text { 5-NN outperforms all other } \\
\text { methods }\end{array}$ & $\begin{array}{l}\text { Future research work can } \\
\text { be focused on Investigation } \\
\text { of the effect of over- } \\
\text { sampling on the k-NN } \\
\text { approach. }\end{array}$ \\
\hline $\begin{array}{l}\text { [18] Bi-Class problem } \\
\text { Ensemble-based } \\
\text { learning algorithm } \\
\text { (DataBoost.IM) }\end{array}$ & 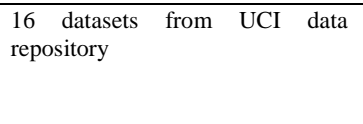 & $\begin{array}{l}\text { C4.5, AdaBoost MI, } \\
\text { DataBoost, } \\
\text { AdaCost, CSB2, } \\
\text { SMOTEBoost }\end{array}$ & $\begin{array}{l}\text { DataBoost.IM outperforms all } \\
\text { other methods }\end{array}$ & $\begin{array}{l}\text { Extend DataBoost.IM for } \\
\text { noisy data, and multi-class } \\
\text { problem. }\end{array}$ \\
\hline $\begin{array}{l}{[19] \text { Multi-class }} \\
\text { problem } \\
3 \text { classifier ( min. } \\
\text { distance, k-NN, and } \\
\text { fuzzy k- nearest })+22 \\
\text { data preprocessing } \\
\text { methods }\end{array}$ & 6 different types of weld flaws & ---- & $\begin{array}{l}\text { 1-NN }+ \text { AHC-KM } \\
\text { preprocessing method is best } \\
\text { suited among all. }\end{array}$ & $\begin{array}{l}\text { Future work can be focused } \\
\text { on development of } \\
\text { evaluation criteria for } \\
\text { multi-class, application of } \\
\text { feature selection together } \\
\text { with data preprocessing } \\
\text { method, and application of } \\
\text { other classifiers on the } \\
\text { same data sets }\end{array}$ \\
\hline
\end{tabular}


Table 2. Performance of classifiers

\begin{tabular}{|c|c|c|c|}
\hline Sampling methods & Classifier & Sensitivity & Specificity \\
\hline \multirow[t]{3}{*}{ SMOTE } & Bayesian & 71.91 & 82. \\
\hline & $\mathrm{k}-\mathrm{NN}$ & 75.06 & 83.2 \\
\hline & SVM & 81.3 & 80.76 \\
\hline \multirow{3}{*}{ LLE+SMOTE } & Bayesian & 72.56 & 83.31 \\
\hline & k-NN & 76.45 & 84.23 \\
\hline & SVM & 84.34 & 83.31 \\
\hline \multirow{3}{*}{$\begin{array}{c}\text { CMTNN (Tech.1)+ } \\
\text { SMOTE }\end{array}$} & $\mathrm{k}-\mathrm{NN}$ & 72.0 & 84.21 \\
\hline & ANN & 77.31 & 85.45 \\
\hline & SVM & 79.0 & 86.41 \\
\hline \multirow{3}{*}{$\begin{array}{c}\text { CMTNN (Tech.2)+ } \\
\text { SMOTE }\end{array}$} & k-NN & 71.4 & 83.01 \\
\hline & ANN & 83.2 & 79.32 \\
\hline & SVM & 84.1 & 79.1 \\
\hline \multirow{3}{*}{$\begin{array}{c}\text { SMOTE+ CMTNN } \\
\text { (Tech.1) }\end{array}$} & $\mathrm{k}-\mathrm{NN}$ & 72.3 & 80.9 \\
\hline & ANN & 85.3 & 84.2 \\
\hline & SVM & 86.01 & 87.5 \\
\hline \multirow{3}{*}{$\begin{array}{c}\text { SMOTE+ CMTNN } \\
(\text { Tech.2) }\end{array}$} & $\mathrm{k}-\mathrm{NN}$ & 82.3 & 85.91 \\
\hline & ANN & 79.4 & 86.01 \\
\hline & SVM & 86.4 & 81.5 \\
\hline
\end{tabular}

\section{REFERENCES}

[1] Xue-wen Chen, Byron Gerlach, and David Casasent, " Pruning support vectors for imbalanced data classification", Proceedings of International Joint Conference on Neural Networks, Montreal, Canada, July 31 - August 4, 2005.

[2] Juanjuan Wang, Mantao Xu, Hui Wang, Jiwu Zhang, "Classification of imbalanced data by using the SMOTE algorithm and locally linear embedding", ICSP2006 Proceedings.

[3] Chao Chen, Andy Liaw, and Leo Breiman, "Using random forest to learn imbalanced data", www.stat.berkeley.edu/tech-reports/666.pdf

[4] Xia Hong, "A kernel-based two-class classifier for imbalanced data sets", IEEE Transactions on Neural Networks, Vol. 18, NO. 1, January2007

[5] Yuchun Tang, Nitesh V. Chawla,, and Sven Krasser, "SVMs modeling for highly Imbalanced classification ", IEEE Transactions On Systems, MAN, and Cybernetics, Vol. 39, No. 1, FEBRUARY 2009

[6] David P. Williams, Vincent Myers, and Miranda Schatten Silvious, "Mine classification with imbalanced data ", IEEE Geoscience and Remote Sensing Letters, Vol. 6, No. 3, July 2009.

[7] Alberto Fernández, María José del Jesus, Francisco Herrera, "Hierarchical fuzzy rule based classification systems with genetic rule selection for imbalanced datasets “, International Journal of Approximate Reasoning 50 (2009) 561-577.

[8] Show-Jane Yen, Yue-Shi Lee, "Cluster-based undersampling approaches for imbalanced data distributions ", Elsevier, Expert Systems with Applications 36 (2009) 5718-5727.

[9] Chao-Ton Su and Yu-Hsiang Hsiao, "An evaluation of the robustness of MTS for imbalanced data ", IEEE Transactions on knowledge and data engineering, Vol. 19, No. 10, October 2007.
[10] Mu-Chen Chen a, Long-Sheng Chen, Chun-Chin Hsu, Wei-Rong Zeng, "An information granulation based data mining approach for classifying imbalanced data ", Elsevier, Information Sciences 178 (2008) 3214-3227.

[11] Jian Huang, L'eon Bottou, C. Lee Giles, "Learning on the border: Active learning in imbalanced data classification “, CIKM'07, November 6-8, 2007, Lisboa, Portugal. ACM 978-1-59593-803-9/07/0011

[12] Son Lam Phung, Abdesselam Bouzerdoum, Giang Hoang Nguyen, "Learning pattern classification tasks with imbalanced data sets ", http://ro.uow.edu.au

[13] Piyasak Jeatrakul, Kok Wai Wong, and Chun Che Fung, "Classification of imbalanced data by combining the complementary neural network and SMOTE algorithm", http://researchrepository.murdoch.edu.au

[14] Yanmin Sun, Mohamed S Kamel, and Yang Wang, "Boosting for learning multiple classes with imbalanced class distribution ", Proceedings of the Sixth International Conference on Data Mining (ICDM'06), 07695-2701-9/06 @ 2006 IEEE.

[15] Chao Chen and Mei-Ling Shyu, "Clustering-based binary-class classification for imbalanced data sets", rvc.eng.miami.edu/paper/2011/IRI11_clustering.pdf

[16] John Doucette and Malcolm I. Heywood, "GP Classification under imbalanced data sets: Active subsampling and AUC approximation“"www.cs.dal.ca/ mheywood/Xfiles/publi cations/JohnEuroGP08.pdf

[17] Zhang and Indrajeet Mani, "kNN approach to unbalanced data distribution: A case study involving information Extraction", Workshop on Learning from Imbalanced Datasets II, ICML, Washington DC, 2003

[18] Hongyu Guo, Herna L Viktor, "Learning from imbalanced data sets with boosting and data generation: The DataBoost-IM approach", Sigkdd Explorations. Volume 6, Issue 1 - Page 30-39 
[19] T. Warren Liao, "Classification of weld flaws with imbalanced class data ", Elsevier, Expert Systems with Applications 35 (2008) 1041-1052

[20] Vladimir Nikulin, Geoffrey J. McLachlan, and Shu Kay $\mathrm{Ng}$, "Ensemble approach for the classification of imbalanced data www.maths.uq.edu.au/ gjm/nmn_lncs09.pdf

[21] Albert Orriols-Puig · Ester Bernadó-Mansilla, "Evolutionary rule-based systems for imbalanced data sets “, Soft Computing (2009) 13:213-225, SpringerVerlag 2008

[22] Jie Gu, "Random Forest Based Imbalanced Data Cleaning and Classification", http://lamda.nju.edu.cn/conf/pakdd07/dmc07/report s/P251.pdf

[23] Bhavani Raskutti, Kovalczyk, "Extreme re-balancing for SVMs: A case Study", Workshop on Learning from Imbalanced Datasets II, ICML, Washington DC, 2003.

[24] Gang Wu, Edward Y. Chang, "Class-boundary alignment for imbalanced dataset learning", Workshop on Learning from Imbalanced Datasets II, ICML, Washington DC, 2003

[25] Mikel Galar, Alberto Fern'andez, Edurne Barrenechea, Humberto Bustince, and Francisco Herrera, "A review on ensembles for the class imbalance problem: Bagging-, Boosting-, and Hybrid-Based Approaches ", IEEE Transactions on systems, MAN, and Cybernetics - 2011.

[26] Giang Hoang NguyenAbdesselam, "Learning pattern classification tasks with imbalanced data sets",

[27] Nitesh V. Chawla, Nathalie Japkowicz, Aleksander Ko lcz, "Editorial: Special issue on learning from imbalanced data sets “, Sigkdd Explorations. Volume 6, Issue 1 - Page 1-6, 2004

[28] Suzan K"oknar-Tezel, Longin Jan Latecki, "Improving SVM classification on imbalanced data sets in distance spaces “, ICDM 2009.

[29] Haibo He, and Edwardo A. Garcia, "Learning from Imbalanced Data “, IEEE transactions on knowledge and data engineering, Vol. 21, No. 9, September 2009

[30] Seyda Ertekin, Jian Huang, C. Lee Giles, "Active learning for class imbalance problem ", www.personal.psu.edu/juh177/pubs/SIGIR2007_sh ort.pdf

[31] Xing-Ming Zhao, Xin Li, Luonan Chen, and Kazuyuki Aihara1," Protein classification with imbalanced data", 2008; 70:1125-1132. VVC 2007

[32] J. Burez, D. Van den Poel, "Handling class imbalance in customer churn prediction ", Expert Systems with Applications 36 (2009) 4626-4636

[33] Baek Hwan Cho, Hwanjo Yu , Kwang-Won Kim , Tae Hyun Kim, In Young Kim, Sun I. Kim, "Application of irregular and unbalanced data to predict diabetic nephropathy using visualization and feature selection methods predict diabetic nephropathy using visualization and feature selection methods ", Elsevier, Artificial Intelligence in Medicine (2008) 42, 37-53
[34] Cheng G. Weng Josiah Poon, "A new evaluation measure for imbalanced datasets ", crpit.com/confpapers/CRPITV87Weng.pdf

[35] Sireesha Rodda, Prof. Shashi Mogalla, "A normalized measure for estimating classification rules for multi-class imbalanced data sets “, International Journal of Engineering Science and Technology (IJEST). 2011

[36] L. Bocchi, G. Coppini, J. Nori, G. Valli, "Detection of single and clustered microcalcifications in mammograms using fractals models and neural networks", Med. Eng. Phys. 26 (4) (2004) 303-312.

[37] Cancer Research UK: Key Facts on Breast Cancer, 2009. http://info.cancerresearchuk.org/cancerstats/types/breast/.

[38] M. De Santo, M. Molinara, F. Tortorella, M. Vento, "Automatic classification of clustered microcalcifications by a multiple expert system", Pattern Recogn. 36(7) (2003) 1467-1477.

[39] I. El-Naqa, Y. Yang, N.P. Galatsanos, et al., "A similarity learning approach to content-based image retrieval: application to digital mammography", IEEE Trans. Med. Imaging 23 (10) (2004) 1233-1244.

[40] I. El-Naqa, Y. Yang, M.N. Wernick, et al., "A support vector machine approach for detection of microcalcifications", IEEE Trans. Med. Imaging 21 (12) (2002) 1552-1563.

[41] J. Ge, B. Sahiner, L.M. Hadjiiski, H.-P. Chan, J. Wei, M.A. Helvie, C. Zhou, "Computer aided detection of clusters of microcalcifications on full field digital mammograms", Med. Phys. 33 (8) (2006) 2975-2988.

[42] L. Hadjiiski, B. Sahiner, H.-P. Chan, et al., "Classification of malignant and benign masses based on hybrid ART2LDA approach”, IEEE Trans. Med. Imaging 18 (12) (1999) 1178-1187.

[43] M. Kallergi, "Computer-aided diagnosis of mammographic microcalcification clusters", Med. Phys. 31 (2) (2004) 314-326.

[44] R. Nakayama, Y. Uchiyama, K. Yamamoto, et al., "Computer- aided diagnosis scheme using a filter bank for detection of microcalcification clusters in mammograms", IEEE Trans. Biomed. Eng. 53 (2) (2006) 273-283.

[45] A. Papadopoulosab, D.I. Fotiadisb, A. Likasb, "Characterization of clustered microcalcifications in digitized mammograms using neural networks and support vector machines", Artif. Intell. Med. 34 (2) (2005) 141-150.

[46] P. Sajda, C. Spence, J. Pearson, "Learning contextual relationships in mammograms using a hierarchical pyramid neural network”, IEEE Trans. Med. Imaging 21 (3) (2002) 239-250.

[47] H. Soltanian-Zadeha, F. Rafiee-Radc, S. PourabdollahNejad, "Comparison of multiwavelet, wavelet, Haralick, and shape features for microcalcification classification in mammograms", Pattern Recognition. 37 (10) (2004) 1973-1986.

[48] J. Ren, D. Wang, J. Jiang, "Effective recognition of MCCs in mammograms using an improved neural classifier", Eng. Appl. Artif. Intell. 24 (4) (2011) 638-645. 
[49] B. Verma, J. Zakos, “A computer-aided diagnosis system for digital mammograms based on fuzzy-neural and feature extraction techniques", IEEE Trans. Inform. Technol. Biomed. 5 (1) (2001) 46-54.

[50] L. Wei, Y. Wei, Y. Yang, R.M. Nishikawab, "Microcalcification classification assisted by contentbased image retrieval for breast cancer diagnosis", Pattern Recognition. 42 (6) (2009) 1126-1132.

[51] L. Wei, Y. Yang, R.M. Nishikawa, et al., "A study on several machine-learning methods for classification of malignant and benign clustered microcalcifications", IEEE Trans. Med. Imaging 24 (3) (2005) 371-380.

[52] D. Soria, J.M. Garibaldi, F. Ambrogi, et al., "A nonparametric version of the Naive Bayes classifier", Knowledge Based System. 24 (6) (2011) 775-784.

[53] M. Salamo, M. Lopez-Sanchez, "Adaptive case-based reasoning using retention and forgetting strategies", Knowledge Based System. 24 (2) (2011) 230-247

[54] Sheshadri H.S, and Kandaswamy A, "Detection of breast cancer tumor based on morphological watershed algorithm”, GVIP, 2005, pp. 17-21.

[55] Sheshadri H.S, and Kandaswamy A, "Experimental investigation on mammogram segmentation for early detection of breast cancer", Journal of Computerized Medical Imaging and Graphics, Elsevier science Vol. 31, 2005, 46-48

[56] Sheshadri H.S. and Kandaswamy A, "Mammogram image analysis using recursive watershed algorithm", National Journal of Technology, Vol. 1, No. 1, 2004, pp. 73-77.

[57] Sheshadri H.S, and Kandaswamy A, "Computer aided decision system for early detection of breast cancer",
Indian Journal of Medical research, Vol. 124, 2006, pp. $149-154$

[58] S. Oporto-D'1az, R. R. Hernandez-Cisneros and H. Terashima-Mar'in, "Detection of microcalcification clusters in mammograms using a difference of optimized Gaussian filters", in Proceedings of the Second International Conference on Image Analysis and Recognition, ICIAR 2005, Toronto, ON, Canada, pp. 998-1005, 2005.

[59] Karssemeijer, N and Hendrikis, L. (1997). Computer assisted reading of mammograms Eur. Radiol. (7), 743 748

[60]Kim, J, K and Park H. W. (1999). Statistical textural features for detection of microcalcifications in digitized mammograms. IEEE Transactions on Medical Imaging (18), 231-238

[61] Mushlin, R and Shapiro, K, D.(1998). Estimating the Accuracy of screening mammography: A meta analysis. Journal of Preventive Medicine vol.14 (2)143-153

[62] Arun kumar M.N and H.S. Sheshadri, "Breast contour extraction and pectoral muscle segmentation in digital mammograms", International Journal of Computer Science and Information Security, Vol 9, No.2, February 2011.

[63] Rolando R. Hern’andez-Cisneros and Hugo Terashima, "Evolutionary Neural Networks Applied To The Classification Of Microcalcification Clusters In Digital Mammograms", 2006 IEEE Congress on Evolutionary Computation Sheraton Vancouver Wall Centre Hotel, Vancouver, BC, Canada July 16-21, 2006 